\title{
Solving another piece of the H5N1 puzzle
}

As the $\mathrm{H} 5 \mathrm{~N} 1$ avian influenza virus continues to sweep across the globe, its potential to emerge as a humanadapted virus remains high. One crucial determinant of the species specificity of influenza viruses is the haemagglutinin (HA) protein: viruses carrying only 3 of the 16 known avian and mammalian HA subtypes (H1,

$\mathrm{H} 2$ and $\mathrm{H} 3$ ) have adapted to humans, but in each case, these viruses have caused major pandemics.

Stevens et al. now report the highresolution crystal structure of the HA of a highly pathogenic H5N1 influenza virus (Viet04 HA). The study published in Science compares the Viet04 HA structure with an avian H5 HA structure (Sing97), and with HA structures from pandemic influenza A viruses - the deadly 1918 human H1 virus and the 1968 human $\mathrm{H} 3$ virus. In addition, the authors use new microarray technology to identify mutations that could allow H5N1 to take hold in the human population.

The general structure of Viet04 HA is similar to other HA structures, consisting of a globular head that contains the receptor-binding domain, a vestigial esterase domain and a membrane-proximal domain. Surprisingly, although the Viet04 HA sequence shows most similarity to that of the avian Sing97 HA, structural comparisons show that Viet04 HA is most closely related to the $1918 \mathrm{H} 1 \mathrm{HA}$. The crystal structure of 1918 HA0 revealed two
$\mathrm{pH}$-sensitive histidine patches, one in the membrane-proximal domain and one in the vestigial esterase domain,

Surprisingly ... Viet04 HA is most closely related to the $1918 \mathrm{H} 1 \mathrm{HA}$. that are thought to promote release of the fusion peptide in the endosome, contributing to pathogenicity. The histidine patch in the membraneproximal domain is conserved in Viet $04 \mathrm{HA}$, and additional structural features in the vestigial esterase might contribute further to virulence.

Using glycan microarray technology, the authors showed that Viet04 HA binds preferentially to $\alpha 2-3$ sialicacid glycan receptors, which typifies the binding specificity of avian influenza viruses. Although mutations in the receptor-binding domain that switch H3 HAs from avian to human specificity did not have a similar effect on Viet04 HA, the binding profile of a double mutant, Gln ${ }^{226} \mathrm{Leu}$, $\mathrm{Gly}^{228}$ Ser Viet04 HA, was markedly altered. This double
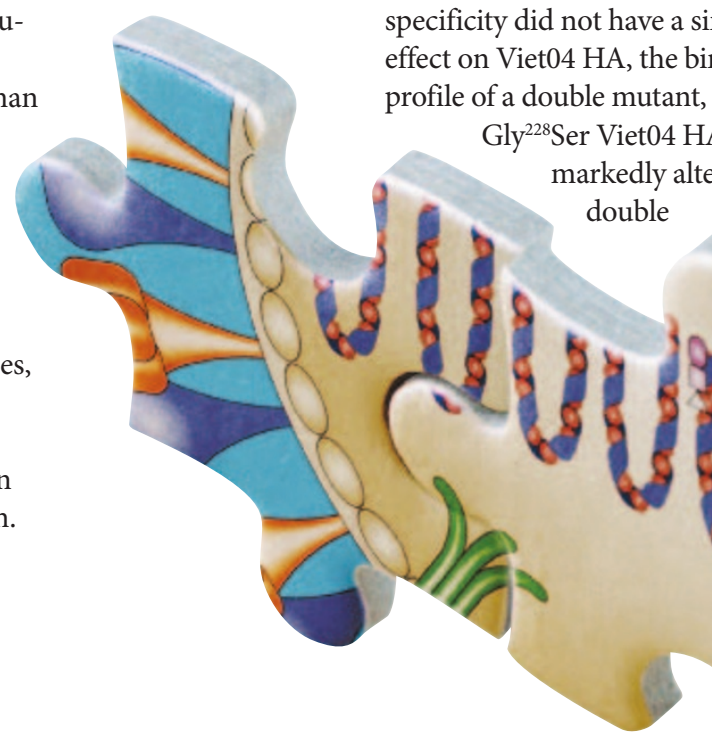

mutant showed reduced affinity for $\alpha 2-3$ receptors, with significant binding to a natural branched $\alpha 2-6$ biantennary glycan. The respiratory mucins in the human upper airway contain $\alpha 2-3$ glycans, which are thought to filter out avian virus that enters the respiratory tract. Reduced binding to these protective mucins coupled with enhanced binding to 22-6 biantennary glycans in the lower airways is one way in which $\mathrm{H} 5 \mathrm{~N} 1$ could establish a foothold in the human host.

Shannon Amoils

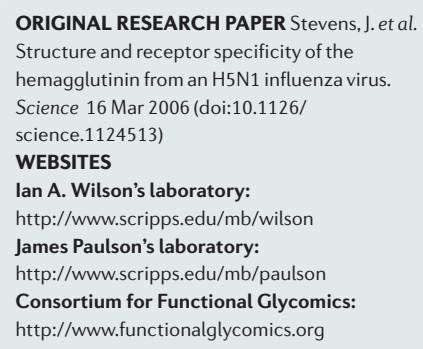

\section{RESEARCH HIGHLIGHTS ADVISORS}

ADRIANO AGUZZI University Hospital of Zürich, Zürich, Switzerland NORMA ANDREWS Yale University School of Medicine, New Haven, CT, USA ARTURO CASADEVALI

The Albert Einstein College of Medicine, Bronx, NY, USA
RITA COLWELL

University of Maryland Biotechnology University of Oxford, Oxford, UK Institute, Baltimore, MD, USA STANLEY FALKOW

Stanford University School of Medicine, Stanford, CA, USA TIMOTHY FOSTER

Trinity College, Dublin, Ireland
KEITH GULL NEIL GOW University of Aberdeen, Aberdeen, UK HANS-DIETER KLENK Philipps University, Marburg Germany
BERNARD MOSS

NIAID, National Institutes of Health, Bethesda, MD, USA JOHN REX

AstraZeneca, Cheshire, UK DAVID ROOS

University of Pennsylvania, Philadelphia, PA, USA 